\title{
Flora y vegetación de suelos crioturbados y hábitats asociados en la Cordillera Blanca, Ancash, Perú
}

\author{
Flora and vegetation of cryoturbed soils and associated habitats in the \\ Cordillera Blanca, Ancash, Peru
}

\begin{abstract}
Asunción Cano ${ }^{1,2}$, Wilfredo Mendoza', Susy Castillo', Marybel Morales', María. I. La Torre $^{1,3}$, Hector Aponte ${ }^{1}$, Amalia Delgado', Niels Valencia ${ }^{1,2}$ y Nanette Vega ${ }^{1}$
\end{abstract}

1 Museo de Historia Natural. Universidad Nacional Mayor de San Marcos (UNMSM). Av. Arenales 1256, Jesús María. 2 Instituto de Investigación de Ciencias Biológicas Antonio Raimondi (ICBAR). UNMSM. Email Asunción Cano: acanoe@unmsm.edu.pe, ashuco@yahoo.com

3 Facultad de Ciencias Naturales y Matemática. Universidad Nacional Federico Villarreal.

Trabajo presentado a la XVIII Reunión Científica del Instituto de Investigaciones en Ciencias Biológicas Antonio Raimondi, "200 años del nacimiento de Charles Darwin y el 150 aniversario de la publicación de On the Origin of Species by Means of Natural Selection". Del 19 al 21 de agosto de 2009 .

Publicado impreso: 20/10/2010 Publicado online: 29/09/2010

\section{Resumen}

Entre los años 2006 y 2008, se llevaron a cabo estudios florístico y de vegetación de los suelos crioturbados y hábitats asociados en cuatro localidades de la Cordillera Blanca (Ancash, Perú) localizadas por encima de los $4500 \mathrm{~m}$. Se realizaron recolectas botánicas además de transectos de intersección-línea, en los cuales se midió el espacio (en $\mathrm{cm}$ ) ocupado por cada especie. Fueron determinadas 136 especies de plantas vasculares, agrupadas en 65 géneros y 26 familias. Las Magnoliópsida (dicotiledóneas) fueron las más diversas con 97 especies, seguidas de las Liliópsidas (Monocotiledóneas) con 36. La mayor diversidad está concentrada en las familias Asteraceae y Poaceae (40,63\%). Los géneros con mayor riqueza de especies fueron Senecio (18) y Calamagrostis (12). Se registraron 76 especies $(54,82 \%)$ en suelos crioturbados y hábitats asociados; mientras que 60 especies $(44,11 \%)$ fueron colectadas en la vegetación adyacente. El 95,56\% de las especies reportadas fueron hierbas perennes. Se caracterizaron cuatro tipos de comunidades vegetales: a) comunidad de suelos crioturbados propiamente dicha, b) comunidad de suelos crioturbados asociada a pajonal, c) comunidad de suelos crioturbados asociada a roquedal seco y d) comunidad de suelos crioturbados asociadas a roquedal húmedo. Se indícan las especies características de cada comunidad.

Palabras claves: flora, suelos crioturbados, Cordillera Blanca, Ancash, Perú.

\section{Abstract}

Since 2006 to 2008 , floristic and vegetational studies on cryoturbed soils and its associated habitats were carried out in four sites above $4500 \mathrm{~m}$, at Cordillera Blanca (Ancash, Peru). Botanical collections and intersection-line transects were made. The space (in $\mathrm{cm}$ ) occupied by each species were measurement. A total of 136 species, in 65 genera and 26 families, were recorded. Magnoliopsida (Dicots) were the most diverse (97 spp.), followed by the Liliopsida (Monocots) (36 spp.). The highest species richness was found in the Asteraceae and Poaceae families (40,63\%). The most diverse genera was Senecio (18) and Calamagrostis (12). We registered 76 species $(54,82 \%)$ in cryoturbed soils and associates habitats, while 60 species $(44,11 \%)$ were recorded for the adjacent vegetation. From the total, $95,56 \%$ of the species were perennials herbs. Four types of plants comunities were characterized: a) community of cryoturbed soil proper, b) community of cryoturbed soil associated with grassland, c) community of cryoturbed soil associated with dry rocky areas, and d) community of cryoturbed soil associated with humid rocky areas. Species associated to each community are given.

Keywords: Flora, Cryoturbed soil, Cordillera Blanca, Ancash, Peru.

\section{Introducción}

La cordillera de los Andes proporciona una gran variedad de hábitats y diversidad biológica al Perú. Entre los ecosistemas andinos de gran interés están la puna y la jalca situados por encima de los $3300 \mathrm{~m}$ de altitud (Young et al. 1997) y el páramo, entre los 3800 y los $4000 \mathrm{~m}$ (Cabrera 1968, Weberbauer 1945).

Suelos crioturbados son aquellos sometidos a una secuencia de hielo y deshielo; fenómeno que ocurre diariamente en la parte alta de los Andes tropicales y que provoca el desplazamiento de partículas, modificando su distribución en las capas del suelo. Esta condición, sumada a las bajas temperaturas, la intensa radiación solar, y otros factores edáficos y climáticos, hacen que la vegetación que colonizan estos suelos sea diferente a la encontrada en hábitats circundantes, principalmente respecto a su diversidad, estructura, fisiología y ecología.

El calentamiento global está afectando actualmente la capa congelada del suelo (permafrost), lo que repercute en la distribución del carbono en el suelo y sus propiedades físicas y químicas (Tarnocai 2002). Estos procesos están muy relacionados a las poblaciones vegetales que habitan estos ambientes, esperando cambios en su composición, adaptaciones y estrategias biogeográficas.

Diversos estudios acerca de estos ambientes han sido realizados en la región Ártica (Gough et al. 2000, Cannone \& Gerdol
2003, Hodkinson et al. 2003, Epstein et al. 2004). En Sudamérica la información disponible se encuentra principalmente referida a las regiones Antártica y Subantártica (Brancaleoni et al. 2003, Gidekel et al. 2003, Bockheim \& Hall 2002). Los Andes son considerados uno de los centros criogénicos más importantes de Sudamérica (Bockheim 2005); a pesar de ello, son escasos los estudios de las comunidades vegetales de estos habitats. Podemos mencionar los de Pavlich y Tovar (1977), que estudiaron la ecomorfología de algunas plantas de la puna, sin hacer énfasis en el tipo de suelos en los que crecían; y recientemente, Tupayachi (2008) que presentó una breve lista de plantas sobre suelos crioturbados, en un estudio sobre la flora de la Cordillera de Vilcanota.

El presente trabajo tuvo como objetivo estudiar la flora y vegetación que se desarrolla en los suelos crioturbados de los Andes centrales del Perú, específicamente en el departamento de Ancash.

\section{Area de estudio}

Durante los años 2006 y 2008 se realizaron recolectas y toma de datos en las siguientes localidades: Punta Olímpica (Asunción), Abra de Cahuish (Recuay), Antamina (Huari) y Abra de Yanashallash (Bolognesi) (Fig. 1, Tabla 1).

1. La Punta Olímpica, a $4900 \mathrm{~m}$, es el abra situada a mayor altitud en la Cordillera Blanca, por donde atraviesa la carretera 

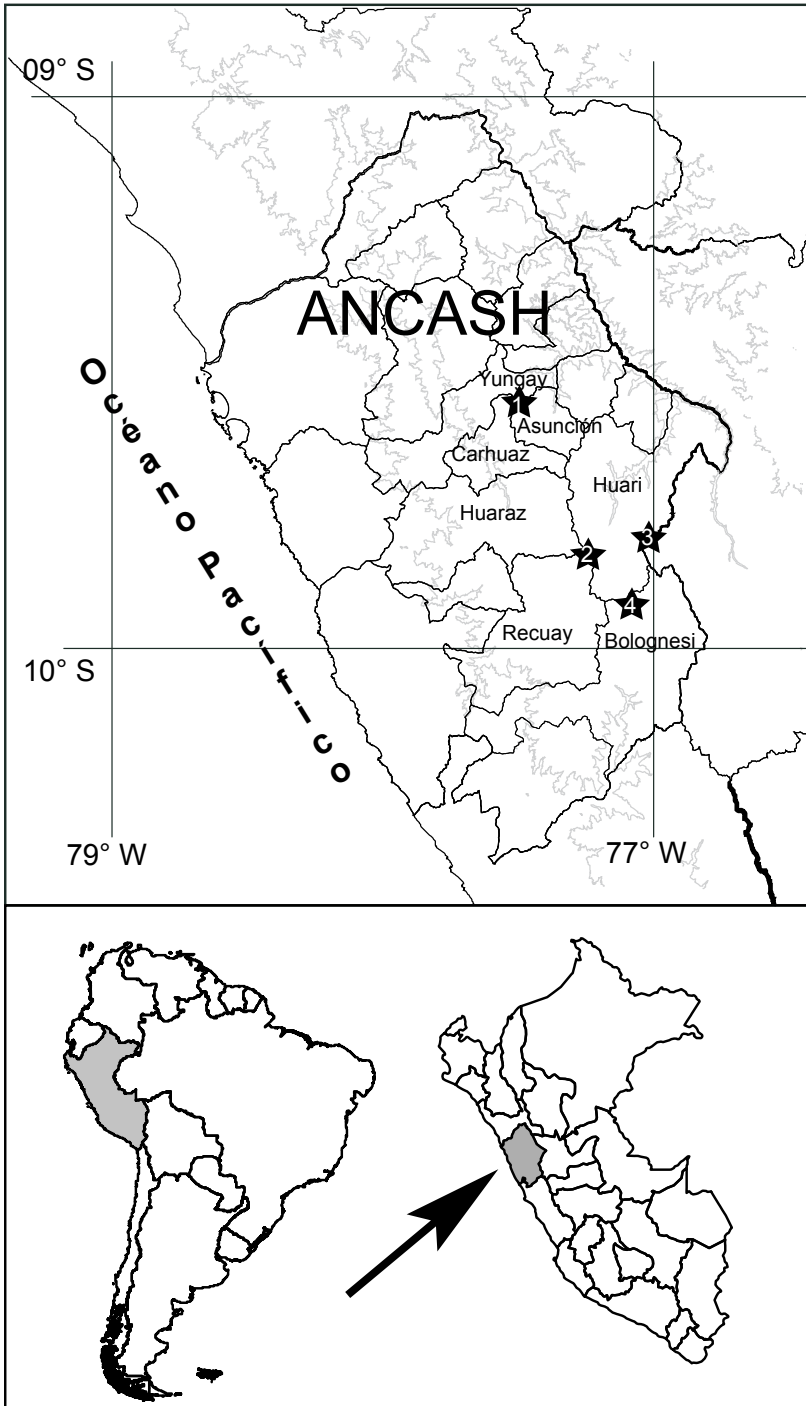

Figura 1. Mapa de ubicación de las localidades de estudio. 1. La Punta Olímpica, 2. El Abra de Cahuish, 3. Antamina y 4. El Abra Yanashallash

que une a la provincia de Carhuaz (Callejón de Huaylas) y Asunción (Callejón de Conchucos). El estudio se realizó en la ladera que pertenece al distrito de Chacas (provincia de Asunción). La zona estudiada se encuentra entre los 4800 y $4940 \mathrm{~m}$, caracterizada por un gran macizo rocoso, con grandes rocas sueltas, piedras y pequeñas áreas de suelo crioturbado. La vegetación es muy rala y frecuentemente asociada a vegetación de roquedales, la que asciende hasta los $5000 \mathrm{~m}$. También hay partes muy húmedas, debido al deshielo, con vegetación semejante a la de oconales (Fig. 2a).

2. El Abra de Cahuish, situado en la provincia de Recuay. Se encuentra atravesado por un túnel que comunica el Callejón de
Huaylas con el de Conchucos (con la provincia de Huari en el extremo sur). La zona de estudio se situó entre los 4500 y 4700m. En esta localidad se pudo encontrar pequeńas extensiones de suelos crioturbados, intercalados con pajonal ralo y algunos arbustos de Loricaria, oconales y vegetación de roquedal, la que asciende por lo menos hasta los $4800 \mathrm{~m}$ (Fig. 2b).

3. Antamina. Esta localidad de muestreo se encuentra en la provincia de Huari (distrito de San Marcos), sobre las cumbres cercanas al campamento de la Compañía Minera Antamina S. A. Las recolectas botánicas y los transectos de evaluación se ubicaron por encima de los $4500 \mathrm{~m}$ en laderas rocoso-pedregosas; intercalados con suelos crioturbados de regular extensión. Estos suelos están rodeados por un pajonal ralo (Fig. 2c). También hay grandes afloramientos rocosos en la cumbre, donde destaca la presencia de Ranunculus macropetalus DC.

4. El Abra Yanashallash, situados entre los distritos de Chiquian y Huallanca, en la provincia de Bolognesi. Los muestreos fueron realizados entre los 4600 y los $4900 \mathrm{~m}$, sobre cumbres, de pendientes moderadas, que presentan las mayores extensiones de suelos crioturbados, intercalados con afloramientos rocosos. Se encuentra un pajonal en la parte inferior y se puede observar plantas aisladas hasta los $4950 \mathrm{~m}$ en los intersticios de las rocas (Fig. 2d).

\section{Metodos}

\section{Estudio de la composición florística}

Para la colecta, herborización y manejo posterior de especimenes de plantas vasculares se emplearon técnicas estándares (Bridson \& Forman 1992). Para determinar la diversidad total se realizó una búsqueda completa en el área de muestreo. La determinación taxonómica de los taxa (familias, géneros y especies) se realizó mediante claves y descripciones disponibles en la literatura botánica, teniendo como base el trabajo de Macbride et al. (1936 y siguientes). También se realizaron consultas en las colecciones del Herbario San Marcos (USM) del Museo de Historia Natural y de otros herbarios nacionales, asi como se recurrió a diversos especialistas en los diferentes taxa.

\section{Estudio de Comunidades Vegetales}

Se realizaron un total de 15 transectos lineales de 50 metros, los cuales fueron ubicados de forma sistemática en las diferentes comunidades de plantas sobre suelos crioturbados. En cada transecto se utilizó el método de intersección - línea, en el cual se registró el espacio (en centímetros) que ocupa una especie determinada en el transecto (Matteucci \& Colma 1982). En los casos en que fue imposible determinar las especies de pastos por la ausencia de floración o por que se encontraban muy mezclados, este conjunto fue registrado como "gramíneas". Los musgos no fueron determinados, sin embargo, fueron registrados en los transectos como "musgos".

Tabla 1. Localidades de estudio, indicando fecha, transectos, rango altitudinal y coordenadas (UTM).

\begin{tabular}{cllccc}
\hline Fechas & Provincia & Localidad & Transectos & Altitud $(\mathbf{m})$ & Coordenadas UTM \\
\hline Mayo 2006 & Huari & Antamina, & $1,2,3$ & $4699-4761$ & $277520,8940386,277317,8936530$ \\
Noviembre 2006 & Recuay & Abra de Cahuish & $4,5,6$ & $4524-4712$ & $252817,8928544,252919,8928758$ \\
Noviembre 2006 & Asunción & Punta Olímpica & 7 & $4800-4940$ & 223917,8989254 \\
Mayo 2008 & Recuay & Abra de Cahuish & $8,9,10$ & $4585-4658$ & $252439,8929656,252632,8928960$ \\
Mayo 2008 & Bolognesi & Abra Yanashallash & $11,12,13,14$ & $4694-4867$ & $272175,8909398,269330,8911400$ \\
Mayo 2008 & Asunción & Punta Olímpica & 15 & $4800-4899$ & 224033,8989170 \\
\hline
\end{tabular}




\section{Análisis de datos}

Los datos fueron colocados en una matriz transecto/especie y analizados mediante un Análisis de Correspondencia (CA). Asimismo se determinó el valor de dominancia y cobertura en cada transecto. Para ello se utilizó el software PAST 1.89 (Hammer et al. 2001).

\section{Resultados}

\section{Diversidad florística}

Se registraron un total de 136 especies de plantas vasculares que crecen en zonas de alta montaña, sobre suelos crioturbados y vegetación asociada (circundante); agrupadas en 65 géneros y 26 familias. Tabla 2 y 3. Los pteridófitos están representados solamente por dos especies en igual número de géneros y familias. Para las gimnospermas, únicamente registramos a Ephedra rupestris Benth. Las magnoliopsidas (dicotiledóneas) fueron el
Tabla 2. Resumen de la diversidad de familias, géneros y especies registradas para los diferentes grupos de plantas vasculares en suelos crioturbados y hábitats asociados en la Cordillera Blanca, Ancash, Perú.

Tabla 3. Familias y especies registradas (en orden alfabético) en el estudio, indicando además la forma de crecimiento, presencia en suelos crioturbados (C) y las registradas en la vegetación asociada, Pa: páramo, Ro: roquedal, RoHu: Roquedal húmedo

\begin{tabular}{|c|c|c|c|c|}
\hline Familia & Especie & $\begin{array}{l}\text { Forma de } \\
\text { crecimiento }\end{array}$ & $\begin{array}{c}\text { Especies } \\
\text { en suelos } \\
\text { crioturbados }\end{array}$ & $\begin{array}{c}\text { Especies } \\
\text { en } \\
\text { vegetación } \\
\text { asociada }\end{array}$ \\
\hline Alstroemeriaceae & Bomarea dulcis (Hook.) Beauverd & Hierba & & $\mathrm{Pa}, \mathrm{Ro}$ \\
\hline Apiaceae & Azorella multifida (Ruiz \& Pav.) Pers. & Hierba & $\mathrm{C}$ & $\mathrm{Pa}, \mathrm{RoHu}$ \\
\hline Apiaceae & Niphogeton scabra (H. Wolff) J.F. Macbr. & Hierba & $\mathrm{C}$ & $\mathrm{Pa}$ \\
\hline Apiaceae & Oreomyrrhis andicola (Kunth) Endl. ex Hook. f. & Hierba & $\mathrm{C}$ & Ro \\
\hline Asteraceae & Baccharis caespitosa (Ruiz E Pav.) Pers. & Subarbusto & $\mathrm{C}$ & $\mathrm{Pa}$ \\
\hline Asteraceae & Belloa longifolia (Cuatrec. \& Aristeg.) Sagást. \& M.O. Dillon & Hierba & $\mathrm{C}$ & $\mathrm{Pa}$ \\
\hline Asteraceae & Chaetanthera cochlearifolia (A. Gray) B.L. Rob. & Hierba & & Ro \\
\hline Asteraceae & Chaetanthera stuebelii Hieron. & Hierba & & Ro \\
\hline Asteraceae & Chersodoma antennaria (Wedd.) Cabrera & Hierba & $\mathrm{C}$ & $\mathrm{Pa}, \mathrm{Ro}$ \\
\hline Asteraceae & Chersodoma deltoidea M.O. Dillon \& Sagást. & Hierba & $\mathrm{C}$ & $\mathrm{Pa}, \mathrm{RoHu}$ \\
\hline Asteraceae & Chersodoma ovopedata (Cuatrec.) Cuatrec. & Hierba & $\mathrm{C}$ & $\mathrm{RoHu}$ \\
\hline Asteraceae & Erigeron sp.1 & Hierba & $\mathrm{C}$ & Ro \\
\hline Asteraceae & Loricaria ferruginea (Ruiz \& Pav.) Wedd. & Arbusto & $\mathrm{C}$ & $\mathrm{Pa}, \mathrm{RoHu}$ \\
\hline Asteraceae & Lucilia kunthiana (DC.) Zardini & Hierba & C & $\mathrm{Pa}, \mathrm{RoHu}$ \\
\hline Asteraceae & Misbrookea strigosissima (A. Gray) V.A. Funk & Hierba & & $\mathrm{RoHu}$ \\
\hline Asteraceae & Mniodes pulvinata Cuatrec. & Hierba & C & Ro \\
\hline Asteraceae & Novenia acaulis (Benth. \& Hook. f. ex B.D. Jacks.) S.E. Freire \& F.H. Hellw. & Hierba & & $\mathrm{Pa}$ \\
\hline Asteraceae & Oritrophium hieracioides (Wedd.) Cuatrec. & Hierba & & Ro \\
\hline Asteraceae & Oritrophium sp.1 & Hierba & $\mathrm{C}$ & $\mathrm{RoHu}$ \\
\hline Asteraceae & Perezia coerulescens Wedd. & Hierba & $\mathrm{C}$ & $\mathrm{Pa}$ \\
\hline Asteraceae & Senecio adenophyllus Meyen \& Walp. & Subarbusto & $\mathrm{C}$ & Ro \\
\hline Asteraceae & Senecio bolivarianus Cuatrec. & Hierba & $\mathrm{C}$ & Ro \\
\hline Asteraceae & Senecio calvus Cuatrec. & Hierba & & Ro \\
\hline Asteraceae & Senecio campanellifer Cuatrec. & Hierba & & Ro \\
\hline Asteraceae & Senecio canescens (Bonpl.) Cuatrec. & Hierba & & $\mathrm{Pa}$, Ro \\
\hline Asteraceae & Senecio comosus Sch. Bip. & Hierba & & Ro \\
\hline Asteraceae & Senecio danai A. Gray & Hierba & $\mathrm{C}$ & Ro \\
\hline Asteraceae & Senecio evacoides Sch. Bip. & Hierba & $\mathrm{C}$ & $\mathrm{Pa}$ \\
\hline Asteraceae & Senecio expansus Wedd. & Hierba & & Ro \\
\hline Asteraceae & Senecio hypsiandinus Cuatrec. & Hierba & $\mathrm{C}$ & Ro \\
\hline Asteraceae & Senecio nivalis (Kunth) Cuatrec. & Hierba & $\mathrm{C}$ & Ro \\
\hline Asteraceae & Senecio nutans Sch. Bip. & Hierba & $\mathrm{C}$ & Ro \\
\hline Asteraceae & Senecio rufescens DC. & Subarbusto & $\mathrm{C}$ & Ro \\
\hline Asteraceae & Senecio sanmarcosensis H. Beltrán & Hierba & & $\mathrm{Pa}$ \\
\hline Asteraceae & Senecio scrobicarioides DC. & Hierba & $\mathrm{C}$ & $\mathrm{RoHu}$ \\
\hline Asteraceae & Senecio serratifolius (Meyen \& Walp.) Cuatrec. & Hierba & $\mathrm{C}$ & $\mathrm{RoHu}$ \\
\hline Asteraceae & Senecio sp.1 & Hierba & $\mathrm{C}$ & $\mathrm{RoHu}$ \\
\hline Asteraceae & Senecio sublutescens Cuatrec. & Hierba & $\mathrm{C}$ & $\mathrm{Ro}, \mathrm{RoHu}$ \\
\hline Asteraceae & Werneria aretioides Wedd. & Hierba & & Ro \\
\hline Asteraceae & Werneria nubigena Kunth & Hierba & $\mathrm{C}$ & $\mathrm{Pa}, \mathrm{RoHu}$ \\
\hline Asteraceae & Werneria pumila Kunth & Hierba & $\mathrm{C}$ & $\mathrm{Pa}$ \\
\hline Asteraceae & Werneria pygmaea Gillies ex Hook. \& Arn. & Hierba & & $\mathrm{RoHu}$ \\
\hline Asteraceae & Xenophyllum dactylophyllum (Sch. Bip.) V.A. Funk & Hierba & $\mathrm{C}$ & $\mathrm{RoHu}$ \\
\hline Asteraceae & Xenophyllum decorum (S.F. Blake) V.A. Funk & Hierba & C & \\
\hline Boraginaceae & Hackelia sp.1 & Hierba & $\mathrm{C}$ & Ro \\
\hline Brassicaceae & Brayopsis alpaminae Gilg \& Muschl. & Hierba & & $\mathrm{Pa}$ \\
\hline Brassicaceae & Brayopsis calycina (Desv.) Gilg \& Muschl. & Hierba & & $\mathrm{Pa}$ \\
\hline Brassicaceae & Brayopsis sp.1 & Hierba & $\mathrm{C}$ & \\
\hline Brassicaceae & Catadysia rosulans O.E. Schulz & Hierba & & Ro \\
\hline Brassicaceae & Descurainia athroocarpa (A. Gray) O. E. Schulz & Hierba & $\mathrm{C}$ & Ro \\
\hline Brassicaceae & Draba argentea O.E. Schulz & Hierba & $\mathrm{C}$ & Ro \\
\hline Brassicaceae & Draba brackenridgei A. Gray & Hierba & & Ro \\
\hline Brassicaceae & Draba cryptantha Hook. f. & Hierba & C & Ro \\
\hline
\end{tabular}

\begin{tabular}{lccc}
\hline Pteridófitos & 2 & 2 & 2 \\
Gimnospermas & 1 & 1 & 1 \\
Magnoliopsidas (dicotiledóneas) & 19 & 47 & 97 \\
Liliopsidas (monocotiledóneas) & 4 & 15 & 36 \\
\hline Total & 26 & 65 & 136 \\
\hline
\end{tabular}


Tabla 3.

\begin{tabular}{|c|c|c|c|c|}
\hline Brassicaceae & Draba ochropetala O.E. Schulz & Hierba & $\mathrm{C}$ & $\mathrm{Pa}$ \\
\hline Brassicaceae & Draba sp. 1 & Hierba & $\mathrm{C}$ & Ro \\
\hline Brassicaceae & Draba sp. 2 & Hierba & & Ro \\
\hline Brassicaceae & Draba sp. 3 & Hierba & & Ro \\
\hline Brassicaceae & Englerocharis peruviana Muschl. & Hierba & & Ro \\
\hline Brassicaceae & Weberbauera sp. & Hierba & & Ro \\
\hline Brassicaceae & Weberbauera spathulifolia (A. Gray) O.E. Schulz & Hierba & & $\mathrm{Pa}, \mathrm{Ro}$ \\
\hline Bromeliaceae & Puya sp. & Subarbusto & & Ro \\
\hline Campanulaceae & Lysipomia sphagnophila Griseb. ex Wedd. & Hierba & & $\mathrm{RoHu}$ \\
\hline Caryophyllaceae & Arenaria boliviana R.O. Williams & Hierba & $\mathrm{C}$ & $\mathrm{RoHu}$ \\
\hline Caryophyllaceae & Paronychia weberbaueri Chaudhri & Hierba & $\mathrm{C}$ & Ro \\
\hline Caryophyllaceae & Plettkea cryptantha Mattf. & Hierba & $\mathrm{C}$ & Ro \\
\hline Caryophyllaceae & Pycnophyllum aschersonianum Muschl. & Hierba & $\mathrm{C}$ & Ro \\
\hline Caryophyllaceae & Pycnophyllum glomeratum Mattf. & Hierba & $\mathrm{C}$ & Ro \\
\hline Caryophyllaceae & Pycnophyllum molle Remy & Hierba & $\mathrm{C}$ & $\mathrm{Pa}, \mathrm{Ro}$ \\
\hline Caryophyllaceae & Silene thysanodes Fenzl & Hierba & $\mathrm{C}$ & Ro \\
\hline Ephedraceae & Ephedra rupestris Benth. & Subarbusto & $\mathrm{C}$ & Ro \\
\hline Ericaceae & Vaccinium floribundum Kunth & Arbusto & & Ro \\
\hline Fabaceae & Astragalus micranthellus Wedd. & Hierba & $\mathrm{C}$ & $\mathrm{Pa}$ \\
\hline Fabaceae & Astragalus uniflorus DC. & Hierba & $\mathrm{C}$ & $\mathrm{Pa}$ \\
\hline Gentianaceae & Gentianella dombeyana (Wedd.) Zaruchhi & Hierba & $\mathrm{C}$ & $\mathrm{Pa}$ \\
\hline Gentianaceae & Gentianella thyrsoidea (Hook.) Fabris & Hierba & & $\mathrm{Pa}$ \\
\hline Gentianaceae & Gentianella weberbaueri (Gilg) Fabris & Hierba & $\mathrm{C}$ & $\mathrm{RoHu}$ \\
\hline Geraniaceae & Geranium jaekelae J.F. Macbr. & Hierba & & $\mathrm{Pa}$ \\
\hline Grammitidaceae & Melpomene flabelliformis (Poir.) A.R. Sm. \& R.C. Moran & Hierba & & Ro \\
\hline Juncaceae & Distichia muscoides Nees \& Meyen & Hierba & & $\mathrm{RoHu}$ \\
\hline Juncaceae & Luzula chilensis Nees \& Meyen ex Kunth & Hierba & & Ro \\
\hline Juncaceae & Luzula racemosa Desv. & Hierba & $\mathrm{C}$ & $\mathrm{Pa}, \mathrm{RoHu}$ \\
\hline Malvaceae & Nototriche antoniana M. Chanco & Hierba & $\mathrm{C}$ & \\
\hline Malvaceae & Nototriche artemisioides Hill & Hierba & $\mathrm{C}$ & Ro \\
\hline Malvaceae & Nototriche coccinea Hill. & Hierba & & Ro \\
\hline Malvaceae & Nototriche pinnata (Cav.) Hill & Hierba & $\mathrm{C}$ & $\mathrm{Pa}, \mathrm{Ro}$ \\
\hline Orobanchaceae & Bartsia adenophylla Molau & Hierba & & Ro \\
\hline Orobanchaceae & Bartsia diffusa Benth. & Hierba & $\mathrm{C}$ & Ro \\
\hline Orobanchaceae & Bartsia elachophylla Diels & Hierba & & Ro \\
\hline Orobanchaceae & Bartsia patens Benth. & Hierba & & Ro \\
\hline Poaceae & Agrostis breviculmis Hitchc. & Hierba & & $\mathrm{Pa}$ \\
\hline Poaceae & Agrostis tolucensis Kunth & Hierba & $\mathrm{C}$ & $\mathrm{Pa}, \mathrm{RoHu}$ \\
\hline Poaceae & Anatherostipa hans-meyeri (Pilg.) Peñailillo & Hierba & & $\mathrm{Pa}$ \\
\hline Poaceae & Bromus lanatus Kunth & Hierba & $\mathrm{C}$ & $\mathrm{Pa}$ \\
\hline Poaceae & Calamagrostis chrysantha (J. Presl) Steud & Hierba & $\mathrm{C}$ & $\mathrm{Pa}, \mathrm{RoHu}$ \\
\hline Poaceae & Calamagrostis eminens (J. Presl) Steud. & Hierba & & $\mathrm{Pa}$ \\
\hline Poaceae & Calamagrostis fuscata (J. Presl) Steud. & Hierba & & $\mathrm{Pa}$ \\
\hline Poaceae & Calamagrostis macrophylla (Pilg.) Pilg. & Hierba & $\mathrm{C}$ & $\mathrm{Pa}$ \\
\hline Poaceae & Calamagrostis minima (Pilg.) Tovar & Hierba & $\mathrm{C}$ & $\mathrm{Pa}$ \\
\hline Poaceae & Calamagrostis nitidula Pilg. & Hierba & $\mathrm{C}$ & $\mathrm{Pa}$ \\
\hline Poaceae & Calamagrostis ovata (J. Presl) Steud. & Hierba & & $\mathrm{Pa}$ \\
\hline Poaceae & Calamagrostis pungens Tovar & Hierba & $\mathrm{C}$ & $\mathrm{Pa}$ \\
\hline Poaceae & Calamagrostis rauhii Tovar & Hierba & $\mathrm{C}$ & $\mathrm{Pa}, \mathrm{RoHu}$ \\
\hline Poaceae & Calamagrostis recta (Kunth) Trin. ex Steud. & Hierba & & $\mathrm{Pa}$ \\
\hline Poaceae & Calamagrostis rigida (Kunth) Trin. ex Steud. & Hierba & $\mathrm{C}$ & $\mathrm{Pa}, \mathrm{RoHu}$ \\
\hline Poaceae & Calamagrostis tarmensis Pilg. & Hierba & $\mathrm{C}$ & $\mathrm{Pa}$ \\
\hline Poaceae & Dielsiochloa floribunda (Pilg.) Pilg. & Hierba & $\mathrm{C}$ & Ro \\
\hline Poaceae & Dissanthelium macusaniense (E.H.L. Krause) R.C. Foster \& L.B. Sm. & Hierba & $\mathrm{C}$ & $\mathrm{Pa}$ \\
\hline Poaceae & Dissanthelium peruvianum (Nees \& Meyen) Pilg. & Hierba & & $\mathrm{Pa}$ \\
\hline Poaceae & Festuca dolichophylla J. L. Presl. & Hierba & $\mathrm{C}$ & $\mathrm{Pa}$ \\
\hline Poaceae & Festuca rigidifolia Tovar & Hierba & & $\mathrm{Pa}$ \\
\hline Poaceae & Festuca subulifolia Benth. & Hierba & & $\mathrm{Pa}$ \\
\hline Poaceae & Festuca weberbaueri Pilg. & Hierba & & $\mathrm{Pa}$ \\
\hline Poaceae & Muhlenbergia peruviana (P. Beauv.) Steud. & Hierba & & $\mathrm{Pa}$ \\
\hline Poaceae & Nassella brachyphylla (Hitchc.) Barkworth & Hierba & & $\mathrm{Pa}$ \\
\hline Poaceae & Poa brevis Hitchc. & Hierba & $\mathrm{C}$ & $\mathrm{Pa}$ \\
\hline Poaceae & Poa gilgiana Pilg. & Hierba & $\mathrm{C}$ & $\mathrm{Pa}$ \\
\hline Poaceae & Poa humillima Pilg. & Hierba & & $\mathrm{Pa}$ \\
\hline Poaceae & Poa pratensis L. & Hierba & $\mathrm{C}$ & $\mathrm{Pa}$ \\
\hline Poaceae & Poa spicigera Tovar & Hierba & & $\mathrm{Pa}$ \\
\hline Poaceae & Trisetum spicatum K. Richt. & Hierba & $\mathrm{C}$ & $\mathrm{Pa}$ \\
\hline Portulacaceae & Calandrinia acaulis Kunth & Hierba & & $\mathrm{Pa}$ \\
\hline Pteridaceae & Eriosorus sp. & Hierba & & Ro \\
\hline Ranunculaceae & Krapfia sp.1 & Hierba & & Ro \\
\hline Ranunculaceae & Ranunculus macropetalus DC. & Hierba & & Ro \\
\hline Rosaceae & Lachemilla frigida (Wedd.) Rothm. & Hierba & & $\mathrm{Pa}$ \\
\hline Rosaceae & Lachemilla pinnata (Ruiz \& Pav.) Rothm. & Hierba & & $\mathrm{Pa}$ \\
\hline Rubiaceae & Galium corymbosum Ruiz \& Pav. & Hierba & & $\mathrm{Pa}$ \\
\hline Saxifragaceae & Saxifraga magellanica Poir. & Hierba & $\mathrm{C}$ & $\mathrm{RoHu}$ \\
\hline Scrophulariaceae & Ourisia muscosa Benth. & Hierba & & RoHu \\
\hline Valerianaceae & Phyllactis rigida (Ruiz \& Pav.) Pers. & Hierba & & $\mathrm{Pa}$ \\
\hline Valerianaceae & Stangea henrici Graebn. & Hierba & $\mathrm{C}$ & \\
\hline Valerianaceae & Valeriana globularis A. Gray & Hierba & & Ro \\
\hline Valerianaceae & Valeriana nivalis Wedd. & Hierba & $\mathrm{C}$ & Ro \\
\hline Valerianaceae & Valeriana sp. 1 & Hierba & $\mathrm{C}$ & Ro \\
\hline Valerianaceae & Valeriana sp. 2 & Hierba & & Ro \\
\hline Valerianaceae & Valeriana weberbaueri Graebn. & Hierba & $\mathrm{C}$ & Ro \\
\hline
\end{tabular}


Tabla 4. Familias con mayor número de géneros y especies en suelos crioturbados y hábitats asociados en la Cordillera Blanca, Ancash, Perú

\begin{tabular}{|c|c|c|}
\hline Familia & Géneros & Especies \\
\hline Asteraceae & 15 & 40 \\
\hline Poaceae & 11 & 31 \\
\hline Brassicaceae & 6 & 15 \\
\hline Caryophyllaceae & 5 & 7 \\
\hline Valerianaceae & 3 & 7 \\
\hline Malvaceae & 1 & 4 \\
\hline Orobanchaceae & 1 & 4 \\
\hline Apiaceae & 3 & 3 \\
\hline Juncaceae & 2 & 3 \\
\hline \multirow[t]{2}{*}{ Gentianaceae } & 1 & 3 \\
\hline & 48 & 117 \\
\hline
\end{tabular}

grupo con mayor riqueza, con 97 especies, distribuidas en 47 géneros y 19 familias. Las familias mas diversas son Asteraceae (15 géneros/ 40 especies), Brassicaceae (6/15) y Caryophyllaceae (5/7). Tabla 4. El género que tiene un mayor número de especies fue Senecio (Asteraceae) con 18 especies. Tabla 5. Para las liliopsidas (monocotiledóneas) registramos 36 especies, en 15 géneros y 4 familias, correspondiendo la mayoría de ellas a las Poaceae (11/31). Los géneros más diversos en esta familia fueron Calamagrostis (12), Poa (5) y Festuca (4) (Tabla 5). Las diez familias con tres o más especies albergaron el 73,84\% al
Tabla 5. Géneros con mayor número de especies en la Cordillera Blanca, Ancash, Perú.

\begin{tabular}{|c|c|}
\hline Géneros & Especies \\
\hline Senecio & 18 \\
\hline Calamagrostis & 12 \\
\hline Draba & 7 \\
\hline Poa & 5 \\
\hline Valeriana & 5 \\
\hline Bartsia & 4 \\
\hline Festuca & 4 \\
\hline Werneria & 4 \\
\hline Brayopsis & 3 \\
\hline Chersodoma & 3 \\
\hline Gentianella & 3 \\
\hline Pycnophyllum & 3 \\
\hline & 71 \\
\hline
\end{tabular}

nivel de géneros y el 86,03\% en especies. Las familias Asteraceae y Poaceae, en conjunto, representaron el 40,63\% (26) del total de géneros reportados y el 52,59\% (71) de las especies (Tabla 3). Los 12 géneros con tres o más especies representan en 52,59\% del total reportado en este estudio (Tabla 5).

De las 136 especie registradas, 76 (56,29\%) están presentes en los suelos crioturbados y muchos de ellos también en la vegetación asociada. Pero solamente cuatro especies (5,26\%) han sido registradas solamente sobre suelos crioturbados. Estas especies son: Stangea henrici Graebn., Xenophyllum decorum (S.F.
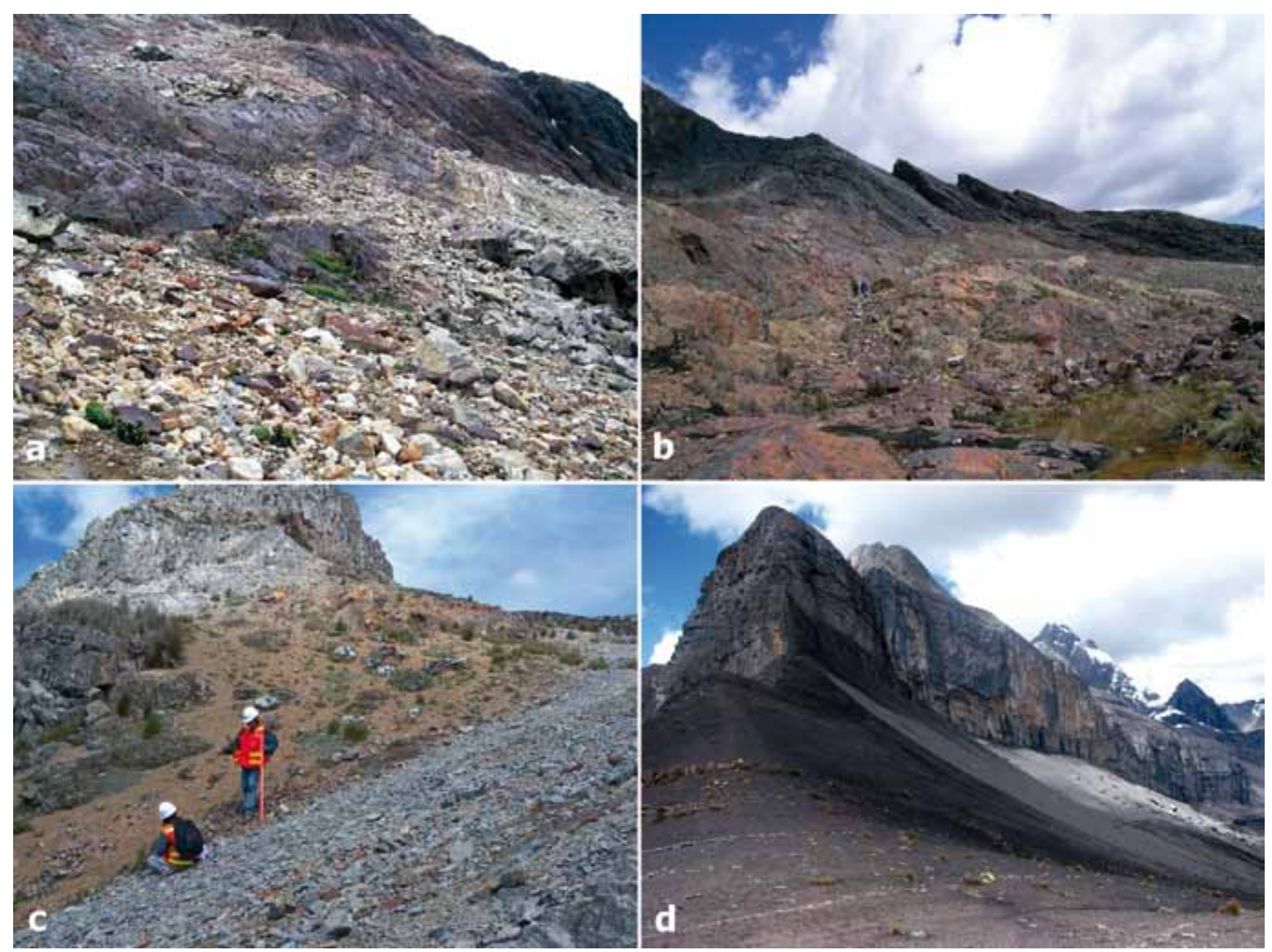

Figura 2. Localidades de estudio: a) Punta Olímpica (Chacas - Asunción), b) Abra de Cahuish (Recuay), c) Antamina (San Marcos - Huari) y d) Abra de Yanashallash (Bolognesi). 


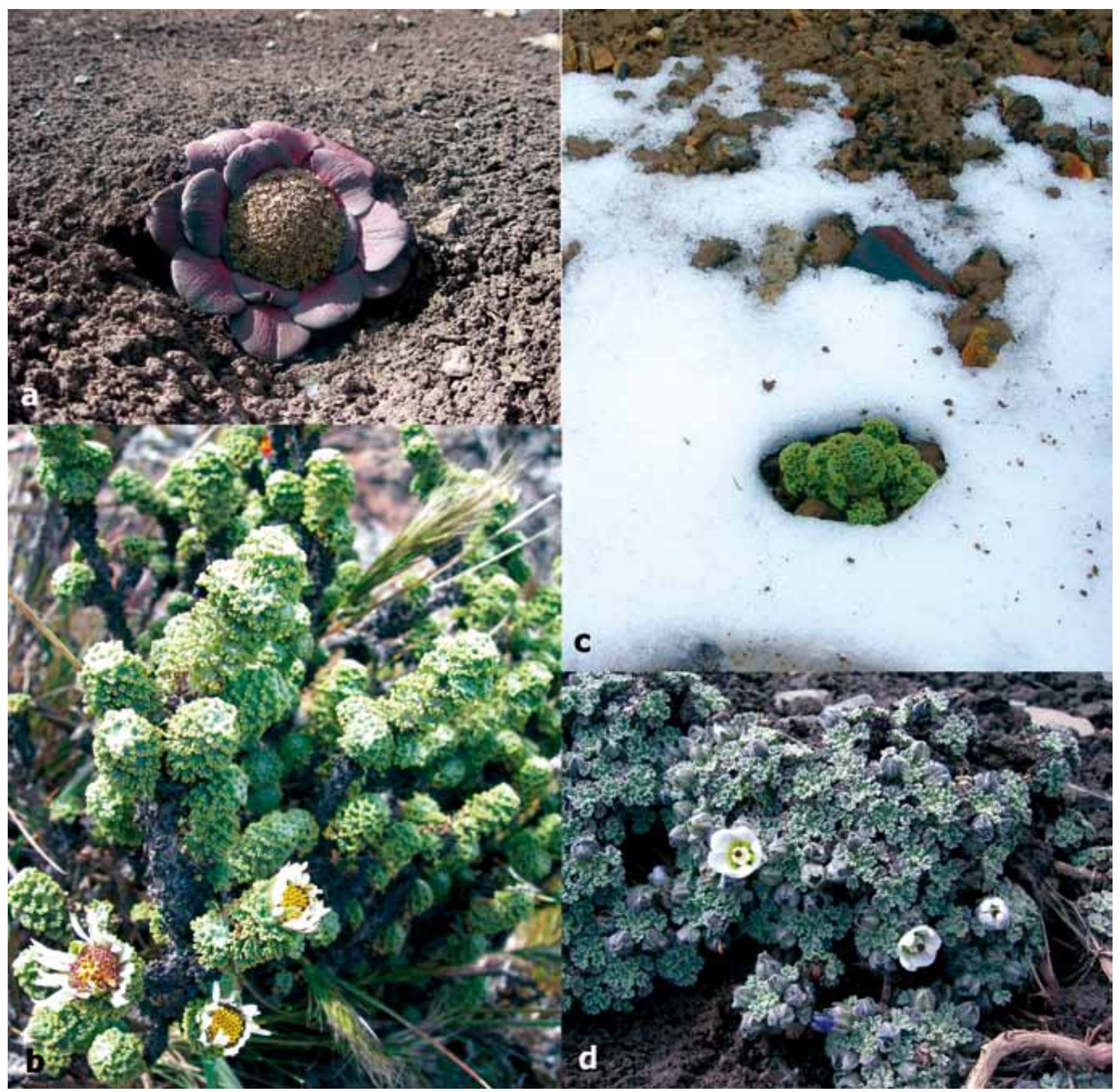

Figura 3. Especies propias de suelos crioturbados: a) Stangea henrici (Valerianaceae), b) Xenophyllum decorum (Asteraceae), c) X. dactyIlophyllum y d) Nototriche antonina (Malvaceae).

Blake) V.A. Funk, Nototriche antoniana M. Chanco, y Brayopsis sp. (Figs. 3a, 3b y 3d). Una especie que estuvo muy bien representada en este tipo de suelos fue Xenophyllum dactylophyllum (Sch. Bip.) V.A. Funk (Fig. 3c), aunque también fue observada en roquedales. Las restantes $60(44,11 \%)$ especies fueron registradas en la vegetación asociada. La vegetación de los roquedales tuvo la mayor diversidad con 55 especies, seguidas por el pajonal con 47 (Tabla 3).

En lo que se refiere a las formas de crecimiento, el $95,56 \%$ de las especies registradas fueron hierbas perennes, el 3,70\% fueron subarbustos y $1,48 \%$ arbustos (S) (Tabla 3).

\section{Comunidades Vegetales}

El análisis de comunidades (Tabla 6) muestra que la cantidad de taxones, la dominancia y la cobertura en cada transecto fueron variables; registrándose hasta 16 taxones por transecto, siendo el transecto 3 (Antamina) el que presentó mayor diversidad. Un
Tabla 6. Número de taxones, cobertura y dominancia en los 15 transectos analizados sobre comunidades vegetales de suelos crioturbados.

\begin{tabular}{cccc}
\hline Transecto & Taxones & \%Cobertura & Dominancia \\
\hline $\mathbf{1}$ & 14 & 61,26 & 0,66 \\
$\mathbf{2}$ & 12 & 62,42 & 0,57 \\
$\mathbf{3}$ & 16 & 18,74 & 0,22 \\
$\mathbf{4}$ & 5 & 6,44 & 0,38 \\
$\mathbf{5}$ & 3 & 5,82 & 0,60 \\
$\mathbf{6}$ & 4 & 4,04 & 0,42 \\
$\mathbf{7}$ & 1 & 8,8 & 1,00 \\
$\mathbf{8}$ & 11 & 24,62 & 0,21 \\
$\mathbf{9}$ & 6 & 7,98 & 0,45 \\
$\mathbf{1 0}$ & 5 & 4,34 & 0,34 \\
$\mathbf{1 1}$ & 6 & 4,94 & 0,24 \\
$\mathbf{1 2}$ & 6 & 5,88 & 0,39 \\
$\mathbf{1 3}$ & 3 & 4,68 & 0,82 \\
$\mathbf{1 4}$ & 5 & 4,84 & 0,73 \\
$\mathbf{1 5}$ & 12 & 10,26 & 0,21 \\
\hline Promedio & 7,27 & 15,67 & 0,48 \\
\hline
\end{tabular}


transecto registró 60 especies lo que representa el 44,12\% del total de especies. En su mayoría los transectos muestran baja dominancia (promedio de dominancia 0,48 ), siendo pocos los transectos de alta dominancia (transectos 7,13 y 14). La cobertura fue igualmente un parámetro variable, en cada transecto siendo en promedio $15,67 \%$ de cobertura para los transectos analizados y llegando a un máximo de $62,42 \%$ de cobertura para el transecto 2 (Antamina).

El resultado obtenido a partir del análisis de correspondencia y las observaciones de campo podemos diferenciar cuatro tipos de comunidades (Fig. 2):

a. Comunidad de crioturbados propiamente dicha: Esta comunidad se caracteriza por encontrase en suelos crioturbados con escasa cobertura vegetal y presentar especies de porte bajo (postradas). Esta compuesta principalmente por especies como Calamagrostis nitidula Pilg., Nototriche antoniana M. Chanco, Poa brevis Hitchc. $y$ Stangea henrici Graebn. Entre las principales especies acompañantes se tiene a Calamagrostis chrysantha (J. Presl) Steud., Descurrainia athroocarpa (A. Gray) O. E. Schulz, Dielsiochloa floribunda (Pilg.) Pilg., Senecio evacoides Sch. Bip., y Senecio rufescens DC. Esta comunidad es la más común y ocupa las mayores extensiones en el Abra de Yanashallash. (Fig. 2d y Fig. 4).

b. Comunidad de crioturbados asociada al pajonal: Esta comunidad se caracteriza por presentar áreas de pajonal de puna y zonas descubiertas (suelos crioturbados), donde la crioturbación se hace más evidente. Las especies más frecuente de esta comunidad son: Calamagrostis pungens Tovar, C. tarmensis Pilger, Festuca dolichophylla J. L. Presl, Gentianella dombeyana (Wedd.) Zaruchhi, Perezia coerulescens Wedd., Pletckea cryptantha Mattf. y Senecio rufescens DC. Esta comunidad está presente en la localidad de Antamina (transectos 1 y 2). (Fig. 2c y Fig. 4).

c. Comunidad de crioturbados asociada a roquedal: Esta es una comunidad que se caracteriza por presentar áreas de suelos crioturbados asociados a suelos rocosos. Las principales especies registradas en esta comunidad son: Baccharis caespitosa (Ruiz \& Pav.) Pers., Dielsochloa floribunda (Pilg.) Pilg., Draba argentea O.E. Schulz, Ephedra rupestris Benth., Niphogeton scabra (H. Wolff) J.F. Macbr., Paronychia Weberbaueri Chaudhri, Senecio nivalis (Kunth) Cuatrec., S. nutans Sch. Bip. y Xenophyllum decorum (S.F. Blake) V.A. Funk. Esta comunidad ha sido registrada en la localidad de Antamina (transecto 3). (Fig. 2c y Fig. 4).

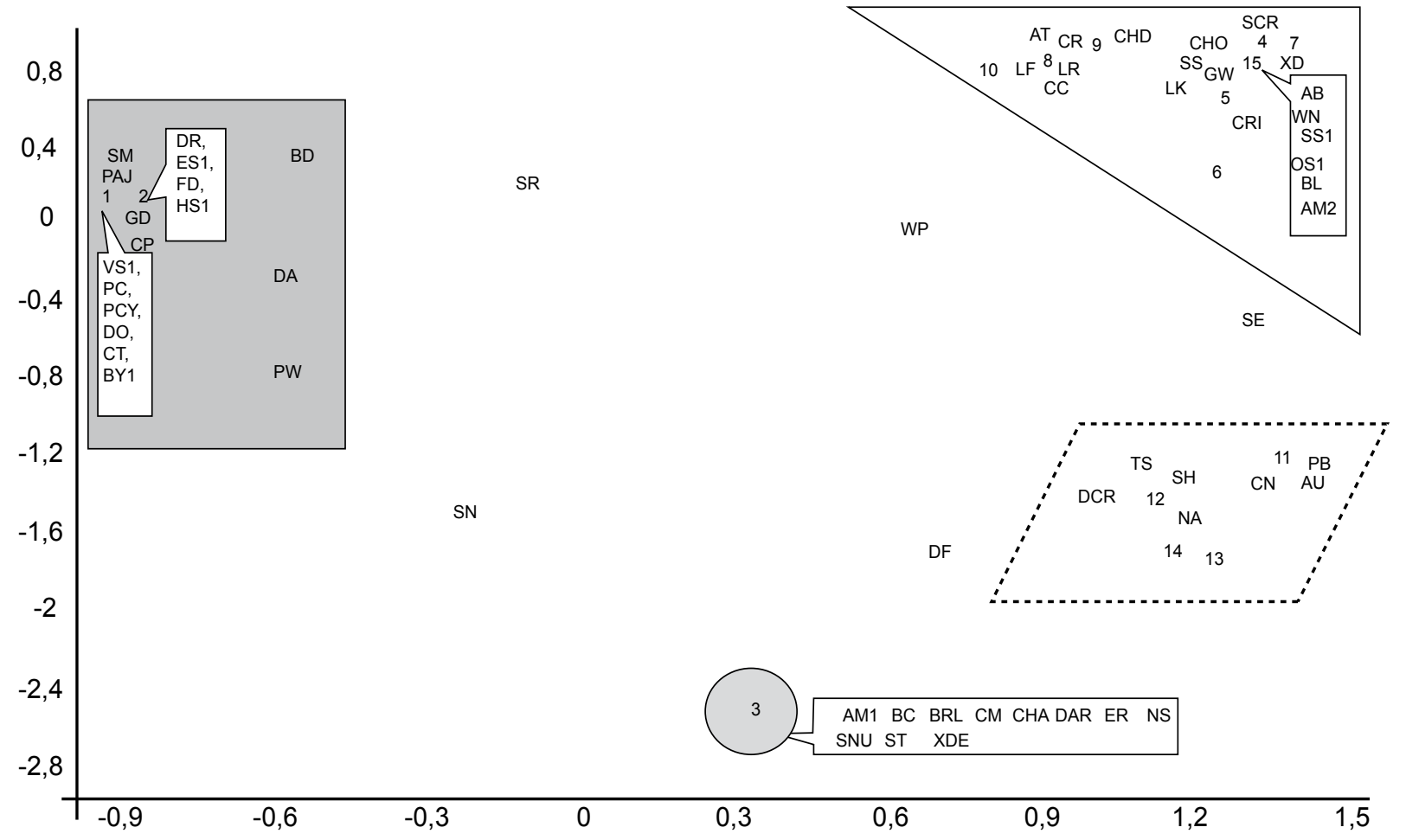

Figura 4. Resultados del análisis de correspondencia utilizando los transectos realizados en las diferentes localidades de estudio. Se aprecian los ejes 1 y 2 por contener la mayor variabilidad. Los números de 1 al 14 corresponden a los transectos realizados en las diferentes localidades. Se aprecian la comunidad de crioturbados asociada al pajonal (rectángulo), comunidad de crioturbados asociado a roquedal (círculo), comunidad de crioturbados propiamente dicha (paralelogramo) y la comunidad de crioturbados asociado a roquedal húmedo (trián-

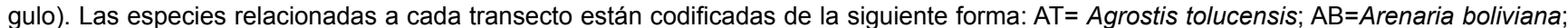
$\mathrm{AM} 1=$ Astragalus micranthellus; $\mathrm{AU}=$ Astragalus uniflorus; $\mathrm{AM} 2=$ Azorella multifida; $\mathrm{BC}=$ Baccharis caespitosa; $\mathrm{BD}=B$ artsia difussa; $\mathrm{BL}=B$ elloa longifolia; $\mathrm{BY} 1=$ Brayopsis sp.1; $\mathrm{BRL}=$ Bromus lanatus; $\mathrm{CC}=$ Calamagrostis chrysantha; $\mathrm{CM}=$ Calamagrostis macrophylla; $\mathrm{CN}=$ Calamagrostis nitidula; $\mathrm{CP}=$ Calamagrostis pungens; $\mathrm{CR}=$ Calamagrostis rauhii; $\mathrm{CRI}=$ Calamagrostis rigida; $\mathrm{CT}=$ Calamagrostis tarmensis; $\mathrm{CHA}=$ Chersodoma antenaria; $\mathrm{CHD}=$ Chersodoma deltoidea; $\mathrm{CHO}=$ Chersodoma ovopedata; $\mathrm{DA}=$ Descurainia athroocarpa; $\mathrm{DF}=$ Delsiochloa floribunda; $\mathrm{DAR}=$ Draba argéntea; $\mathrm{DCR}=$ Draba cryptantha; $\mathrm{DO}=$ Draba ochropetala; $\mathrm{DR}=$ Draba sp.1; $\mathrm{ER}=$ Ephedra rupestris; $\mathrm{ES} 1=$ Erigeron sp.1; $\mathrm{FD}=$ Festuca dolichophyIla; GD=Gentianella dombeyana; GW=Gentianella weberbaueri; HS1=Hackelia sp1; LF=Loricaria ferruginea; LK=Lucilia kunthiana; LR=Luzula racemosa; NS=Niphogeton scabra; NA=Nototriche antoniana; OS1=Oritrophium sp1; PW=Paronichia weberbaueri; $\mathrm{PC}=$ Perezia coerulescens; $\mathrm{PCY}=$ Plettkea cryptantha; $\mathrm{PB}=$ Poa brebis; $\mathrm{SM}=$ Saxifraga magellanica; $\mathrm{SE}=$ Senecio evacoides; $\mathrm{SN}=$ Senecio nivalis; SNU=Senecio nutans; $\mathrm{SR}=$ Senecio rufescens; $\mathrm{SCR}=$ Senecio scrobicarioides; SS1=Senecio sp.1; SS=Senecio sublutescens; $\mathrm{ST}=$ Silene thysanodes; $\mathrm{SH}=\mathrm{Stangea}$ henrici; TS=Trisetum spicatum; VS1=Valeriana sp.1; WN=Werneria nubigena; WP=Weneria pumilla; XD=Xenophyllum dactylophyllum; $\mathrm{XD}=$ Xenophyllum decorum. 


\section{d. Comunidad de crioturbados asociada a roquedal húme-}

do: Esta comunidad se caracteriza porque los suelos crioturbados alternan o son contiguos a zonas rocosas de mayor humedad, debido al deshielo y las filtraciones. En los roquedales húmedos, debido a la presencia constante de agua, se forma una vegetación a manera de un "micro-oconal". En esta comunidad las especies más frecuentes son: Agrostis toluscensis Kunth, Calamagrostis chrysantha (J. Presl) Steud, C. rauhii Tovar, C. rigida (Kunth) Trin. ex Steud., Chersodoma deltoidea M.O. Dillon \& Sagást., Ch. ovopedata (Cuatrec.) Cuatrec., Dielsochloa floribunda (Pilg.) Pilg., Gentianella weberbaueri (Gilg) Fabris, Luzula racemosa Desv., Senecio rufescens DC., S. sublutescens Cuatrec. y Xenophyllum dactylophyllum (Sch. Bip.) V.A. Funk. Esta comunidad ha sido reportada para las localidades de Punta Olímpica y Abra de Cahuish (Figs. 2A, 2B, y Fig. 4).

\section{Discusión y conclusiones}

El presente estudio reporta un total de 136 especies de plantas vasculares, creciendo en suelos crioturbados y vegetación asociada, en altitudes por encima de los $4500 \mathrm{~m}$. Siendo aun escasos los estudios, en el Perú, que aborden en forma particular la flora y vegetación de las altas cumbres, consideramos que los resultados son un aporte importante y referente para otros estudios. Particularmente en el caso de la vegetación en suelos crioturbados, nuestro estudio es prácticamente es el pionero a nivel de país, ya que no solo hemos evaluado la composición florística, sino que se ha caracterizado la vegetación mediante un análisis cuantitativo. Reportamos 76 especies de plantas vasculares que crecen en suelos crioturbados; de las cuales solo cuatro especies se pueden considerar propias o exclusivas de este hábitat; las demás especies (72) ocurren en uno o más tipos de la vegetación asociada. El número de especies registradas en el presente trabajo en suelos crioturbados en la Cordillera Blanca (Ancash) es bastante mayor a las 18 especies reportadas por Tupayachi (2005) para la Cordillera de Vilcanota (Cusco), para este mismo hábitat. La diferencia encontrada entre ambas localidades pueden deberse a varios factores, entre los que se puede señalar la distribución latitudinal de las especies, el factor geológico y el diseño del estudio. Ambas floras comparten siete especies: Ephedra rupestris, Xenophyllum dactylophyllum, Senecio canescens, Draba cryptantha, Calamagrostis ovata, Saxifraga magellanica y Valeriana nivalis. Pero entre estas especies compartidas no se encuentra a ninguna de las cuatro especies que reportamos como exclusivas de suelos crioturbados.

También nuestros resultados confirman un patrón biogeográfico para la flora altoandina, como es la importancia de algunas familias. Gentry (1993) mencionó que las Asteraceae (20\%) y Poaceae $(7-14 \%)$ son la familias dominantes y más especiosas sobre el límite superior de los bosques; así mismo señaló que más de la mitad de las especies de las floras altoandinas perteneden a las 10 familias más ricas en especies. En este estudio reportamos la presencia de 40 especies de Asteraceae y 31 de Poaceae, que representan el 29,42\% y 22,79\%, respectivamente; además entre ambas familias representan el 52,59\% del total reportado. Así mismo las 10 familias más especiosas en nuestro estudio, presentan el 86,03\% del total de las especies.

Respecto a la vegetación, con los resultados obtenidos, ha sido posible apreciar la variabilidad de las comunidades de acuerdo a la zona de colecta y a las características del área. De las cuatro localidades estudiadas, se han logrado identificar cuatro comuni- dades distintas, siendo el Abra de Cahuish y Punta Olímpica las localidades que presentaron mayor similitud en la composición de especies de los transectos. Se trata de comunidades que en su mayoría ocupan áreas reducidas, por la difíciles condiciones topográficas. El muestreo en más localidades permitirá conocer mejor la estructura de las comunidades en suelos crioturbados.

Con el estudio de transectos podemos confirmar algunas de las características fisionómicas que poseen las comunidades en suelos crioturbados. Tanto la cobertura como el número de especies son bajas en las comunidades de suelos crioturbados propiamente dichos, y aumentan de forma significativa en los transectos cercanos al pajonal, roquedal y roquedal húmedo.

Los recientes cambios climáticos podrían ocasionar la pérdida del ciclo hielo - deshielo, lo cual afectaría el ciclo de nutrientes. Algunos estudios muestran que este tipo de cambios en el ciclo de nutrientes favorece la presencia de algunas especies, teniendo consecuencias sobre la estructura de las comunidades de estas zonas. Es así que, la dinámica de estas comunidades se convierte en un indicador potencial de cambios ambientales (Kelley \& Epstein, 2009) y son un factor a considerar en futuros estudios en zonas altoandinas.

Concluimos que la vegetación sobre suelos crioturbados presenta una diversidad, dominancia y cobertura baja, con solamente cuatro especies que consideramos exclusivas este tipo de hábitats, las demás especies son propias de la vegetación asociada al pajonal, roquedal y roquedal húmedo

\section{Agradecimientos}

Expresamos nuestro reconociendo al Vicerrectorado de Investigación de la Universidad Nacional Mayor de San Marcos por el apoyo económico a través de los Estudios de Investigación 061001011 y 081001161 . También expresamos nuestra sincera gratitud a nuestro colega José Roque por la preparación del mapa de la zona de estudio y las figuras; a Mónica Arakaki, Blanca León y Kenneth R. Young por la revisión del manuscrito y sus valiosas sugerencias al mismo.

\section{Literatura citada}

Bockheim, J. G. \& K. J. Hall. 2002. Permafrost, active-layer dynamics and periglacial environments of continental Antarctica. South African Journal of Science 98, January/ February 2002. pp:82-90

Bockheim, J. G. 2005. Final Report, International Workshop on Antarctic Permafrost and Soils. November 14-18, 2004, University of Wisconsin, Madison, WI. Office of Polar Programs, Antarctic Section. National Science Foundation.

Brancaleoni, L., J. Strelin \& R. Gerdol. 2003. Relationships between geomorphology and vegetation patterns in subantartic Andean tundra of Tierra del Fuego. Polar Biology 26: 404-410.

Bridson, D. \& L. Forman. 1992. Herbarium Handbook. Royal Botanical Gardens, Kew. p 303.

Cabrera, A. L. 1968. Ecología vegetal de la puna. In Troll, C. (ed.), Geo-Ecology of the mountainous region of the tropical Andes. Colloquium Geographicum 9, Bonn. Pp. 91-116.

Cannone, N. \& R. Gerdol. 2003. Vegetation as an ecological indicator of surface inestability in rock glaciers. Artic, Antartic, and Alpine Research, 35(3): 384-390.

Epstein, H. E., J. Beringer, W. A. Gould, A. H. Lloyd, c. D. Thompson, F. S. Chapin III, G. J. Michaelson, C. L. Ping, T. S. Rupp \& D. A. Walker. 2004. The nature of spatial transitions in The Artic. Journal of Biogeography 31: 1917-1933. 
Gentry, A. H. 1993. Overview of Peruvian Flora. In Brako, L. \& J. Zarucchi, Ctalogue of Flowering Plants and Gymnosperns. Missouri Botanical Garden, Mongraphs in Systematic Botany 45: 29-39.

Gidekel, M., L. Destéfano-Beltrán, P. García, L. Mujica, P. Leal, M. Cuba, L. Fuentes, L. A. Bravo, L. J. Corcuera, M. Alberdi, I. Concha \& A. Gutierrez. 2003. Extremophiles 7: 459-469.

Gough, L., G. R. Shaver, J. Carroll, D. L. Royer \& J. A. Laundre. 2000. Vascular plant species richness in Alaskan artic tundra: the importance of soil $\mathrm{pH}$. Journal of Ecology $88,54-66$.

Hammer, Ø., D. A. T. Harper, \& P. D. Ryan, 2001. PAST: Paleontological Statistics Software Package for Education and Data Analysis. Palaeontologia Electronica 4(1): 9pp. http:// palaeo-electronica.org/2001_1/past/issue1_01.htm

Hodkinson, I. D., S. J. Coulson \& N. R. Webb. 2003. Community assembly along proglacial chronosequences in the high Artic: Vegetation and soil development in north-west Svalbard. Journal of Ecology 91: 651-663.

Kelley, A. \& H.E. Epstein. 2009. Effects of Nitrogen Fertilization on Plant Communities of Nonsorted Circles in Moist Nonacidic Tundra, Northern Alaska. Arctic, Antarctic, and Alpine Research 41(1):119-127.
Macbride, J. F. et al. 1936 y siguientes. Flora of Peru. Field Museum of Natural History, Botanical Series, Chicago.

Moore, P.D. \& Chapman, S.B. 1986. Methods in Plant Ecology, 2nd ed. Blackwell Scientific Publications, Oxford.

Matteucci, S. D. y A. Colma. 1982. Metodología para el estudio de la vegetación. Organización de los Estados Americanos, Serie de Biología, Monografía No 22. pp.168.

Pavlich, M. \& O. Tovar. 1977. Ecomorfología de algunas plantas de la puna del Perú central. Archivos de Biología Andina 7(1): 27-53.

Tarnocai, C. 2002. Cryosols in a changing environment: their role and research needs. 17th World Congress on Soil Science, 14-21 Agosto 2002, Tailandia. Simposio No.43, 86-1:86-6.

Tupayachi, A. 2005. Flora de la Cordillera Vilcanota. Arnaldoa 12. (1-2): $126-144$.

Weberbauer, A. 1945. El Mundo Vegetal de los Andes Peruanos. Ministerio de Agricultura, Lima.

Young, K. R., B. León \& A. Cano. 1997. Peruvian Puna. In S. D. Davis, V. H. Heyewood, O. Herrera-Macbride, J. Villa-Lobos and A. C. Hamilton (Eds.), Centres of Plant Diversity. A Guide and Strategy for their Conservation. Volume 3, The Americas. The World Wide Fund and IUCN-The World Conservation Union. 470-476. 


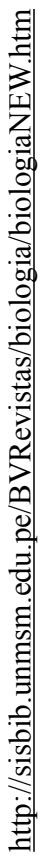

